\title{
Staat und Agrarsektor in West-Malaysia ${ }^{1}$
}

\author{
Von Volker Kasch
}

\section{Zur Rolle des Staates in Malaysia}

In der entwicklungstheoretischen und -politischen Diskussion über Fragen der Abhängigkeit der Länder der Dritten Welt von den Metropolen der Weltgesellschaft, ihrer Armut und Unterentwicklung sowie von Möglichkeiten und Strategien zu ihrer Überwindung ist in wachsendem Maße die Rolle des Staates im Entwicklungsprozeß der Peripherieländer in den Blickpunkt des Forschungsinteresses gerückt. (Hanisch/Tetzlaff 1981) Auf empirischer Ebene läßt sich deutlich ein beträchtlicher Funktionszuwachs des Staates in der Dritten Welt ablesen. Ein wachsendes Maß an Staatsintervention erfolgt einerseits über die Herausbildung eines staatskapitalistischen Sektors und andererseits über die Steuerung und Lenkung des Akkumulationsprozesses mit Hilfe spezifischer Industrialisierungs- und Agrarpolitiken. Sowohl aufgrund seiner dominanten Rolle als ökonomischer Akteur als auch als Institution politischer Herrschaft, die ihre Position und Stabilität im Spannungsfeld von Repression und Legitimation (Elsenhans 1976) einschließlich ideologischer Momente sichert, bildet der Staat eine wesentliche Determinante des gesellschaftlichen Entwicklungsprozesses in der Dritten Welt. Der Staat kann jedoch weder als ein Instrument begriffen werden, das einerseits beliebig einsetzbar ist, und der andererseits über einen eigenen, genuinen Handlungsspielraum verfügt, noch kann ihm a priori eine progressive soziale Funktion zugesprochen werden, sondern er ist vielmehr als Konkretion der divergierenden und konkurrierenden Interessen der gesellschaftlich dominanten Klasse anzusehen, als deren Ausdruck entsprechend auch seine Entwicklungspolitik zu analysieren ist.

Mit der Konzipierung der New Economic Policy (NEP) und ihrer Implementierung seit Beginn des Second Malaysia Plans (SMP/1971-75) tritt der malaysische Entwicklungsweg in seine zweite Phase ein, die sich deutlich von der ersten postkolonialen 'MerdekaPhase unterscheidet. Der malaysische Staat übernimmt zusätzliche Funktionen, indem er mit Hilfe des Instruments der Staatsbetriebe als eigenständiger Akteur im Akkumulationsprozeß auftritt. Er greift damit erstmals in den Industrie- und Handelssektor ein, der bis zur NEP ausschießliches Aktionsfeld des inländischen (chinesischen), aber insbesondere des Auslandskapitals war, das 1970 63,3 \% des Produktionsvermögens im scorporate sector' (Malayen: 2,4\% und Nichtmalayen/Chinesen: 34,3\%) kontrollierte (Third Malaysia Plan/TMP: 86).

1 Erweiterte Fassung eines Vortrages auf der wissenschaftlichen Fachtagung der Deutschen Gesellschaft für Asienkunde in Königstein/Taunus, 26. April 1981. Auf eine ausführliche Quellenangabe wurde hier verzichtet; vgl. dazu Kasch 1982. 
Dieser Funktionswandel des malaysischen Staates in der zweiten Phase des Entwicklungsweges ist direkter Ausdruck eines nach den Mai-1969 Unruhen eingetretenen Transformationsprozesses der Herrschaftsstruktur, in der als Folge eines internen Herrschaftskonflikts die malayische bürokratische Elite durch eine entstehende, kapitalistisch orientierte Staatsklasse abgelöst wurde (Jomo 1978). Im Gegensatz zum Industriesektor war die NEP nicht mit einer grundsätzlichen Neuorientierung der Agrarpolitik verbunden. Denn sie war seit Erlangung der nationalstaatlichen Souveränität immer schon interventionistisch gegenüber einem Laissez - faire der Industrialisierungspolitik. Während die Staatsklasse im Industrie- und Handelssektor eigene Interessen und Politikziele wie die Umverteilung der Anteile am Produktivvermögen des 'corporate sectors zugunsten der malayischen Ethnie nach dem Schlüssel Malayen: 30 \%, Nichtmalayen: $40 \%$ und Ausländer: $30 \%$ durchzusetzen versucht, mit der sie sich über einen expandierenden Staatssektor letztlich eine eigene ökonomische Basis schafft, ist die Agrarpolitik, mit deren Hilfe in der Hauptsache das zweite Ziel der NEP: seradication of poverty irrespective of racer erreicht werden soll, ein wesentliches Instrument, um einerseits das Wirtschaftswachstum zu forcieren und um andererseits das aus der absoluten Armut der Bauern resultierende Konfliktpotential abzumildern und ihre sozio-ökonomische Integration zu gewährleisten. Denn letztlich nur ihre Loyalität garantiert auch die Stabilität der bestehenden Herrschaftsstruktur. Da die NEP in erster Linie auf den städtisch-industriellen Sektor zielt, erhält die Agrarpolitik somit eine auf mehreren Ebenen wichtige flankierende Funktion. Unter dieser politischen neben der entwicklungspolitischen Fragestellung soll im folgenden untersucht werden, welche Ziele der Staat im Agrarsektor verfolgt, welche Instrumente er eingesetzt hat und welche Effekte sie einerseits für die soziale Lage der Bauern gehabt haben und, andererseits, ob das politische Ziel der Sicherung und Legitimierung der Herrschaftsposition erreicht wurde.

\section{Soziopolitische Determinanten der Agrarpolitik}

Der malaysische postkoloniale Staat orientierte sich in seiner Wirtschafts- und Entwicklungspolitik zumindest in der ersten Phase des Entwicklungsweges grundsätzlich am ordnungspolitischen Status quo, so daß eine Abkehr vom sozioökonomischen Strukturmuster der Kolonialzeit nicht stattfand, z. B. durch die Nationalisierung des Primärsektors oder den Einsatz des Instruments der Agrar- oder Landreform. Auf politischer Ebene waren derartige drastische Strukturreformen durch die Notwendigkeit beschränkt, die kommunalistische Balance des mit Erlangung der nationalstaatlichen Souveränität im Jahre 1957 im Rahmen der Allicance-Koalition geschlossenen Herrschaftsbündnisses der jeweiligen Elite der malayischen (53\%), chinesischen (35\%) und indischen (11\%) Bevölkerungsgruppe aufrechtzuerhalten. Dieser basierte auf dem Kompromiß, nicht in die jeweiligen Interessensphären einzugreifen, allein unter der Einschrän-

2 Im Jahre 1963 wurde unter Einschluß der ehemaligen britischen Kolonialgebiete Sarawak, Sabah und Singapore die Federation of Malaysia gebildet, aus der Singapore im Jahre 1965 wieder ausschied. 
kung, die politisch qua Verfassungsbestimmungen privilegierte Position der Malayen (Special Rights) zu respektieren und insgesamt ihre ökonomisch inferiore Stellung zu verbessern. Für die Aufrechterhaltung des Status quo war nicht zuletzt die dominante Rolle des Auslandskapitals ausschlaggebend. Seine Interessen als externer Akteur der Herrschaftsallianz bildeten neben der politischen Hegemonie der Malayen den zentralen Bestimmungsfaktor der Politik des postkolonialen Staates.

Während die chinesische Elite als Interessenvertreter der chinesischen Bourgeoisie und der kleinen Händler fungierte, repräsentierte die malayische bürokratische Elite zunächst sich selbst, war jedoch auf die Masse der malayischen Bauern als politischer Basis angewiesen, da ihr eine ökonomische fast vollständig fehlte. Der aus diesem politischen Strukturmuster resultierende spezifische Legitimationszwang der malayischen Elite gegenüber den Bauern erhält seinen besonderen Akzent aufgrund der Tatsache, daß die malayische Regierungspartei United Malayan National Organization (UMNO) von Anfang an mit der konservativen islamisch orientierten Partai Islam (PI) konkurrierte, die für sich ebenfalls die Rolle des originären Repräsentanten der malayischen Bauernschaft reklamierte. In Kelantan, dem ärmsten Bundesstaat auf der Halbinsel mit über 90 \% malayischem Bevölkerungsanteil war die PI auch entsprechend erfolgreich (Kessler 1978) und konnte sich dort von 1959 bis 1978 in der Regierung behaupten. Sowohl die Notwendigkeit der Sicherung der Massenloyalität der malayischen Bauern, die zugleich die Legitimationsfähigkeit der malayischen Herrschaftsstruktur insgesamt widerspiegelt, als auch der Parteienkonflikt um ein identisches Wählerpotential bilden daher eine wesentliche Determinante der Entwicklungspolitik des malaysischen Staates. Entsprechend der politischen Hegemonie der malayischen Elite (UMNO) sowie der Interessen des Auslandskapitals besitzt der Agrarsektor eindeutige Priorität im Rahmen staatlicher Entwicklungspolitik, was im Gegensatz zur Praxis der überwiegenden Zahl der Peripheriegesellschaften steht.

\section{Konfliktfeld: Ländliche Armut}

Das erstmals für das Jahr 1970 mit Daten dokumentierte Ausmaß der absoluten Armut in Malaysia kennzeichnet deutlich das soziale Problemfeld der Agrarpolitik. Spätere Untersuchungen belegten, daß im Zeitraum von 1957-70 die Realeinkommen des Großteils der ländlichen Bevölkerung drastisch gesunken waren, während in Durchschnittswerten gemessen die Realeinkommen insgesamt einen Anstieg aufwiesen (Lee 1977). Zusätzlich besitzt das Armutsproblem seine politische Brisanz in dem Umstand, daß die Konzentration der absoluten Armut im Agrarsektor mit einem Anteil von 73,6\% der Gesamthaushalte die malayischen Haushalte besonders trifft, da sie $83 \%$ der Kleinproduzentenhaushalte stellen. Mit seiner spezifischen ethnischen Komponente beinhaltet das Armutsproblem stets ein latentes politisches Konfliktpotential von erheblichem Ausmaß.

Von der Hälfte der malaysischen Haushalte (49,3\%), die 1970 in absoluter Armut lebte, befand sich mit knapp drei Vierteln $(73,6 \%)$ ein weit überproportionaler Anteil im 
Agrarsektor. 68,3\% der landwirtschaftlichen Haushalte verfügten nicht über ein Einkommen, das ihnen die Befriedigung ihrer Grundbedürfnisse, so die Armutsdefinition des Third Malaysia Plans, ermöglichte gegenüber 27,8\% der nichtlandwirtschaftlichen Haushalte. Die Differenzierung der Armutsgruppen nach ihrer ökonomischen Tätigkeit zeichnet folgendes Bild: Mit 28,6\% aller Armutshaushalte bilden die Kautschukkleinproduzenten die absolut größte Armutsgruppe. Addiert man noch die Haushalte der Plantagenarbeiter hinzu, so zeigt sich, daß die Hälfte aller Armutshaushalte im Agrarsektor aus der Exportproduktion ihr Einkommen bezieht. Während die exportorientierte Kautschukproduktion auf der einen Seite Wachstumsmotor der malaysischen Ókonomie ist, liegt auf der anderen Seite die Kehrseite der Medaille in der absoluten Armut eines Großteils der Produzenten. Neben der übrigen Landwirtschaftı (91,8\%) ist die Gruppe der Reisbauern mit einem Anteil von 88 \% (TMP: 163) am stärksten betroffen. Hier handelt es sich fast ausschließlich um malayische Haushalte $(97 \%)$. Zusammengenommen entfallen allein auf die Kautschuk- und die Reisproduktion zwei Drittel aller armen Haushalte. In diese beiden Bereiche fließt entsprechend auch ein Großteil der staatlichen Entwicklungsausgaben neben der Neulanderschließung als dem Großprojekt der malaysischen Agrarpolitik.

\section{Technokratisch-bürokratischer Charakter der Agrarpolitik}

Das soziale Konfliktfeld der ländlichen Armut impliziert, daß die Agrarpolitik des malaysischen Staates eine Doppelfunktion zu erfüllen hat. Erstens hat sie unter Aufrechterhaltung der weltmarktabhängigen Akkumulationsstruktur das Wachstum und die Modernisierung des Exportsektors zu fördern, um damit gleichzeitig die Durchkapitalisierung und die Kommerzialisierung des gesamten Agrarsektors voranzutreiben. Zweitens übernimmt sie zusätzlich die politische Funktion, die soziale Integration der bäuerlichen Armutsgruppen zu gewährleisten, damit dieses Konfliktpotential sich nicht herrschaftsbedrohend für die malayische Elite entlädt. Da die stets latente Brüchigkeit der kommunalistischen Herrschaftsstruktur in Zeiten sich verschlechternder realer Lebensbedingungen der Landbevölkerung aktualisiert wird, was sich in den Wahlergebnissen von 1959 und 1969 oder in den Hungermärschen der Bauern von Baling 1974 und jüngst in den Demonstrationen der Reisbauern von Kedah im Januar 1980 niedergeschlagen hat, ist die politische Kontrolle der Landbevölkerung die notwendige Voraussetzung für die Stabilität der bestehenden Herrschaftsstruktur. Die politische Kontrolle, gleichbedeutend mit Depolitisierung der Bauern, ist der politische Imperativ, der als Grundmuster alle Agrarentwicklungsprojekte des malaysischen Staates durchzieht. Sie wirkte nicht nur restriktiv hinsichtlich jeglicher Form der Partizipation und der Selbsthilfe der Bauern (z. B. im Bereich des Genossenschaftswesens), sondern führte zu einer politischen Bürokratisierung und Zentralisierung von 'Rural Development' (Fredericks 1977), die neben dem wachstums- und modernisierungstheoretischen 'Betting on the Strong als 'Top-Down-Planning' oder als 'Reform From Above' charakterisiert werden muß. 
Bezeichnendes Beispiel dafür ist der Versuch des Landwirtschafts- und Genossenschaftsministers Aziz bin Ishak zu Anfang der 60er Jahre das zwischen malayischen Reisproduzenten und den chinesischen Händlern bestehende Ausbeutungsverhältnis der Distributionssphäre durch die Úbertragung eines Vermarktungsmonopols an lokale Genossenschaften zu durchbrechen. Dieser Reformversuch scheiterte am Widerstand der Minister der Malaysian Chinese Association (MCA), die die Interessen der chinesischen Kleinhändler vertraten, mit der Folge, dal? der populäre Aziz bin Ishak einige Zeit später aus der Regierung entfernt wurde. Auch bei der Diskussion um die Ausgestaltung der NEP wurde die Errichtung von staatlichen Vermarktungsunternehmen für Agrarprodukte, wie sie von dem ansonsten recht einflußreichen Prof. Ungku Aziz vorgeschlagen wurde, von vornherein abgeblockt (Vorys v. 1975: 405).

Lagen Reformmaßnahmen einerseits nicht im Interesse einer der Fraktionen der herrschenden Klasse, so wurde auf der anderen Seite auch von den Bauern kein entsprechender direkter Druck ausgeübt. Bauernbewegungen oder politisch aktive Genossenschaften sind in Malaysia nicht existent. Als ein wesentlicher Erklärungsfaktor für die fehlende Organisation der Bauern muß das vorherrschende ethnische Bewußtsein angesehen werden, d. h. der Kommunalismus überlagert die Klassengegensätze und verhindert die Perzeption von Konflikten in sozialen Kategorien der Ungleichheit ökonomischer Strukturen (Kasch 1982: Kap. 4.5).

Die Organisationsstruktur der staatlichen Land Settlement Schemes verhindert zudem die gewerkschaftliche Organisation der Siedler. Aber auch die von Indern dominierte National Union of Plantation Workers (NUPW) hat sich in ihrer Politik als äußerst systemloyal gezeigt.

Die verstärkte Intervention des Staates im malaysischen Akkumulationsprozeß mit der Implementierung der NEP ist für den Agrarsektor neben der Expansion von Staatsunternehmen im Agrobusiness, hauptsächlich mit der Errichtung zahlreicher staatlicher und halbstaatlicher Entwicklungsbehörden auf Bundes- und Staatenebene (Development Authority) verbunden. Sie bilden jetzt das wichtigste Instrument der Agrarpolitik des Staates, zumal sie mit über zwei Dritteln im TMP der bedeutendste Empfänger der staatlichen Entwicklungsausgaben sind. Mit der steigenden Zahl der Entwicklungsinstitutionen (80) wurde zwar eine Spezialisierung hinsichtlich der jeweiligen Servicefunktion erzielt, diese geht jedoch einher mit einer Bürokratisierung, die dem Staat eine direkte Einflußnahme und Kontrolle über den Agrarsektor ermöglicht. Dies geschah u. a. bei der Zwangsintegration der Farmer's Associations - außer in der MUDA-Region - in der Farmer's Organisation Authority (FOA), in der Selbstorganisation durch zentralisierte Bürokratie ersetzt wurde.

\section{Die Ziele der Agrarpolitik}

Die agrarpolitischen Ziele beschränkten sich fast ausschließlich auf die Förderung des Wachstums der Agrarproduktion und die horizontale Diversifizierung der monokulturellen Produktstruktur. Während die Wachstumspolitik im Exportsektor über die Er- 
schließung von Neuland und die Produktivitätssteigerung qua Modernisierung des Kautschukbaumbestandes auf die Steigerung der Exporterlöse zielte und gleichzeitig zusätzliche Arbeitsplätze in den Land Development Schemes schaffen sollte, war sie im Nahrungsmittelsektor auf die Importsubstitution von Reis gerichtet. Mit Hilfe einer steigenden Reisproduktion sollte die extrem hohe Importabhängigkeit (1957: $46 \%$ ) bis zum Erreichen der vollständigen Selbstversorgung reduziert werden. Hinter diesem Ziel stand sowohl das Motiv der Devisenersparnis als auch wesentlich das der Sicherung der Nahrungsmittelversorgung, um eventuellen Krisensituationen vorzubeugen. Damit verbunden war die wachsende Kommerzialisierung der Reisproduktion und damit auch die Marktintegration der Reisbauern, die bei den Kautschukbauern bereits gegeben war. Zusätzlich wird ein steigender Grad der Weiterverarbeitung der eigenen Rohstoffe angestrebt, ${ }^{3}$ um die bestehenden komparativen Vorteile zu dynamisieren und die achtziger Jahre sind zur 'Decade of Resouce-Based Industriesı deklariert worden.

Direkte einkommenspolitische Ziele fehlten vollständig, da die Einkommensentwicklung als abhängige Variable des Wirtschaftswachstums begriffen wurde. Verteilungspolitische Maßnahmen widersprachen zudem der grundsätzlichen ordnungspolitischen Orientierung der staatlichen Politik. Dies änderte sich erst mit dem im Rahmen der NEP formulierten Ziel der Umverteilung des Produktivvermögens im 'corporate sector', das jedoch in keiner Weise die Entwicklung der Individualeinkommen und insbesondere nicht der Bauernschaft positiv beeinflussen kann, da sie von der Umverteilung faktisch ausgeschlossen sind. Das zweite Ziel der NEP: reradication of poverty irrespective of racer enthält zwar ein verbales politisches Bekenntnis, ist aber nicht mit der Implementation direkter Instrumente zur Einkommenssteigerung der Bauern verbunden.

\section{Staatliche Agrar-Entwicklungsausgaben}

Die entscheidende Voraussetzung für eine aktive Agrarpolitik des malaysischen Staates bildete ein ausreichender finanzieller Handlungs- und Verteilungsspielraum, den hohe reale Wirtschaftswachstumsraten des BSP (1970-78: ca. $8 \%$ ) schufen, die wesentlich von einer günstigen Entwicklung der Terms of Trade insbesondere seit Mitte der siebziger Jahre getragen wurden und von denen auch der Staat einen beträchtlichen Anteil absorbierte. Absolut konnte er seine Einnahmen von 1969-78 vervierfachen und im gleichen Zeitraum seinen relativen Einkommensanteil bezogen auf das BSP von $19 \%$ auf $25,1 \%$ steigern, wozu nicht zuletzt die enorm gestiegenen Einnahmen aus der Erdölproduktion beitrugen. Im Gegensatz zum Großteil der Peripherieländer profitierte Malaysia dank seiner Erdölressourcen von der Preispolitik der OPEC. Die Ausdehnung der Finanzbasis des Staates fand seinen direkten Niederschlag in den Volumina der Entwicklungspläne. So wurden die Ausgaben vom First Malaysia Plan (1966-70) auf den Second Malaysia Plan (1971-75) mehr als verdoppelt (4,2-9,8 Mrd. M\$). Für den Third Malay-

3 So soll z. B. Palmöl ausschließlich im weiterverarbeiteten Zustand exportiert werden. 
sia Plan (1976-80) war zunächst ein Finanzrahmen von 18,5 Mrd. M\$ projektiert worden, was ebenfalls einer knapp hundertprozentigen Steigerungsrate entsprach, die aber im Mid-Term-Review (MTR) noch einmal um weitere 13,5 Mrd. M\$ nach oben revidiert wurde. Diese drastische Steigerung der staatlichen Entwicklungsausgaben entsprang dem ökonomischen Erfordernis, den erheblichen Ausfall an Privatinvestitionen zu kompensieren, um die angestrebten Wachstumsziele zu erreichen. Denn ein stiller Investitionsboykott bildete die passive Form des Widerstands der chinesischen Bourgeoisie gegen die Verteilungspolitik der NEP. Der Mid-Term-Review wies einen deutlichen Rückgang der privaten Ersparnisse sowie der Investitionen aus, während gleichzeitig der Kapitalabfluß ins Ausland mit 7,5 Mrd. M\$ die dreifache Höhe des Planwertes erreicht hatte (MTR-TMP: 119).

In der sektoralen Allokation der staatlichen Entwicklungsausgaben liegt die eindeutige Priorität auf dem Agrarsektor, zumal ein Großteil der Infrastrukturausgaben ebenfalls im ländlichen Raum getätigt wurden, so daß z. B. im Rahmen des Second Five Year Plan (1961-65) über $55 \%$ der Gesamtentwicklungsausgaben auf den ländlichen Sektor entfielen. Die herausragende Rolle des Agrarsektors in der Entwicklungspolitik des malaysischen Staates zeigt sich insbesondere im Vergleich mit dem Industrie- und Handelssektor, für den im First Malaysia Plan (1966-70) lediglich 3,3 \% der Gesamtausgaben alloziert wurden, als deutlicher Reflex einer zumindest bis zu diesem Zeitpunkt nicht existenten aktiven importsubstituierenden Industrialisierungspolitik. Sie war einerseits durch die Interessen des britischen Handelskapitals und andererseits durch die Tatsache blockiert worden, daß die Förderung der industriellen Entwicklung zur Kapitalakkumulation der chinesischen Beourgeoisie beigetragen hätte, wodurch die bereits bestehende ethnisch ungleiche Verteilung des Produktivvermögens noch verstärkt worden wäre.

Ein gravierender Einschnitt wurde erst mit der Implementation der NEP vollzogen, als im Second Malaysia Plan (1971-75) die Ausgaben für den Industrie- und Handelssektor um das Fünffache auf $16,5 \%$ gesteigert wurden. Im TMP wurden sie jedoch wieder auf 9,5\% zurückgeschraubt, während der Agrarsektor seinen Anteil wieder um $4 \%$ auf 25,5\% steigern konnte. Dort zeigt sich eine deutliche Konzentration der Mittelverwendung auf drei Bereiche: die Neulanderschließung, die seit 1956 über die Hälfte der gesamten Agrarentwicklungsausgaben absorbierte, die Modernisierung des Kautschukbaumbestandes und Bewässerungsprojekte im Reisanbau. Die Aufteilung der Entwicklungsausgaben zwischen dem exportorientierten und dem nahrungsmittelproduzierenden Sektor ist ausgehend vom zweiten Fünfjahresplan (1961-65) mit ca. $42 \%$ für den Nahrungsmittelsektor bis heute konstant geblieben. Nur im ersten Malaysia-Plan (1966-70) stieg er auf über die Hälfte ( $51 \%)$ an, weil in dieser Periode die beiden großen Bewässerungsprojekte MUDA in Kedah/Perlis und KEMUBU in Kelantan in Angriff genommen wurden. Diese Zahlen indizieren, daß die Förderung des agrarischen Exportsektors zumindest nicht auf Kosten der Nahrungsmittelproduktion erfolgte, sondern diese einen fast gleichgewichtigen Ausgabenanteil erhielt. Bezogen auf die Flächen- oder Beschäftigtenanteile war dieser sogar weit überproportional. Dennoch muß die Frage gestellt werden, ob diese zwar massiven Finanzmittel ausreichend sind, die Armut der malaysischen 
Bauern aber ohne strukturelle Reformmaßnahmen hinsichtlich der Bodenbesitzverteilung zu überwinden?

\section{Die Struktur des Agrarsektors}

Zum Zeitpunkt der Unabhängigkeit (1957) dominierte der Agrarsektor und speziell sein exportorientiertes Segment die malaysische Okonomie und damit den gesamten Reproduktionskreislauf, der auf dem Export von Primärprodukten (einschließlich Zinn) und dem Import von Konsum- und Investitionsgütern basierte, da die eigene Industriestruktur erst recht rudimentäre Züge aufwies. Der Agrarsektor erwirtschaftete 43,3\% des BIP, zwei Drittel der Exporterlöse und absorbierte knapp $60 \%$ der Beschäftigten. Nicht zuletzt aber bildeten die Exportsteuern mit die wichtigste Einnahmequelle des Staates. Neben seiner Größe und entsprechenden Bedeutung waren die vorhandenen komparativen Kostenvorteile der Agrarexportproduktion ausschlaggebend für die führende Rolle des Agrarsektors in der weltmarktintegrativen Entwicklungsstrategie Malaysias.

Ein übergewichtiger Exportsektor prägt die malaysische Agrarstruktur. Allein $70 \%$ der bewirtschafteten Gesamtfläche von ca. 8 Mill. acres (1975) wird von den beiden Hauptexportprodukten Kautschuk (52\%) und Olpalmen (18\%) in Anspruch genommen. Von ihm abhängig und funktional auf ihn bezogen erfolgte die Entwicklung des Nahrungsmittelsektors mit Reis als Hauptanbauprodukt, dessen Anteil an der kultivierten Agrarfläche bei ca. $12 \%$ liegt. Hier hat sich der Prozeß der Kommerzialisierung fast vollständig durchgesetzt, so daß eine ausschließliche Reproduktion auf Subsistenzbasis in WestMalaysia kaum noch anzutreffen ist.

Wesentliches Charakteristikum des bis zur Einführung der Olpalme monokulturell auf Kautschuk ausgerichteten Exportsektors ist die Koexistenz eines vom britischen Kapital dominierten Plantagen- mit einem Kleinproduzentensektor. Dieser etablierte sich gegen den massiven Widerstand des Kolonialstaates, der einerseits die Interessen der Plantagenbesitzer vertrat, andererseits die Nahrungsmittelversorgung sichern wollte, indem er die malayischen Bauern in der Rolle von Reisproduzenten zurückerhalten wollte, obgleich die Kautschukproduktion auch bei niedrigsten Weltmarktpreisen stets komparative Einkommensvorteile gegenüber der Reisproduktion aufwies (Lim Teck Ghee 1977). Die Existenz eines ausgedehnten Plantagensektors impliziert die frühzeitige Genese eines ruralen Proletariats, die jedoch in der Hauptsache nicht über die Freisetzung von malayischen Bauern erfolgte, sondern auf dem Wege der Immigration, d. h. des Imports der benötigten Ware Arbeitskraft aus Indien und China. ${ }^{4}$ Somit wurde die soziale Transformation Malaysias in eine Klassengesellschaft durch die Implantierung der kapitalistischen Produktionsweise wesentlich auch durch diesen exogen induzierten Proletarisierungsprozeß bestimmt.

Das herausragende Spezifikum der malaysischen Agrarentwicklung liegt in der Verfüg-

4 Die Domäne der Chinesen in dem sich in der Kolonialökonomie herausbildenden ethnisch segmentierten, sektoralen Spezialisierungsmuster bildete jedoch der Zinnbergbau; vgl. dazu Kasch 1982: Kap. 2.3. 
barkeit eines beträchtlichen Landpotentials. Nach Schätzungen läßt sich die bereits kultivierte Agrarfläche West-Malaysias von 8 Mill. acres (1975) noch um weitere 6 Mill. acres ausdehnen. Mit der Kultivierung der tropischen Urwaldgebiete, d. h. Inwertsetzung der ökonomisch wertvollen Ressource Land, befindet sich Malaysia in der günstigen Lage, den Wachstumsprozeß z. T. nicht durch eine konfliktträchtige Reallokation knapper Ressourcen bewerkstelligen zu müssen, sondern bisher ungenutztes Land sowie die Arbeitskraft unterbeschäftigter oder landloser Bauern produktiv in den gesellschaftlichen Reproduktionsprozeß i. S. des 'vent for surplus' eingliedern zu können.

Im Zeitraum von 1957-75 wurde die agrarisch genutzte Fläche durch private aber in der Hauptsache durch staatliche Neulanderschließung um ca. 3 Mill. acres erweitert. Seit 1920 hat sich die kultivierte Gesamtfläche verachtfacht. Während lediglich 200000 acres Neufläche für die Reisproduktion verwandt wurden, schluckte der Exportsektor den Löwenanteil, von dem 500000 acres auf Kautschuk und weitere 1,3 Mill. acres auf die im Rahmen der Diversifizierungspolitik neu eingeführte Olpalme entfielen. In den letzten Jahren konnten auch Kakao, Zuckerrohr, Kaffee und Tabak ihre Flächen ausdehnen. Hinsichtlich der Landgröße und der Organisationsform läßt sich der Agrarsektor in folgende drei Bereiche aufgliedern: Plantagen mit einer Betriebsgröße über 100 acres, die zumeist als Limited Companies organisiert sind; Kleinproduzenten, die eine Fläche unter 100 acres bewirtschaften und Land Settlement Schemes staatlicher Entwicklungsgesellschaften.

\subsection{Plantagen}

Knapp ein Drittel (30 \%) der kultivierten Agrarfläche der malaysischen Halbinsel wurde 1975 von 2500 Plantagen in Anspruch genommen: Kautschuk (1814), Olpalme (627), Kokosnuß (39) und Tee (20), auf denen knapp 250000 Plantagenarbeiter beschäftigt waren. Im Zeitraum von 1957-75 stagnierte die Plantagenfläche mit einem minimalen Flächenzuwachs von $10 \%$ gegenüber zwei Dritteln der Gesamtfläche. Die Ursachen dieser relativen flächenmäßigen Stagnation lagen zum einen in der restriktiven Landvergabepolitik des Staates, der die Neulanderschließung in eigener Regie übernahm und zum anderen in einem Disengagement der Plantagen im Kautschuksektor. Ende der fünfziger Jahre wurden 230000 acres (ca. 12 \% der Plantagenfläche) in einem Prozeß, der als subdivision of estates bezeichnet wurde, in kleine Parzellen aufgesplittert. Zwar reduzierte sich der Anteil der Plantagen an der Kautschukfläche von $54 \%$ auf $33 \%$, aber dieser Rückgang wurde durch den Wechsel auf die weitaus profitablere Olpalme kompensiert, wodurch zugleich ein Diversifizierungseffekt erzielt wurde. Die Konversion von der Kautschuk- zur Palmölproduktion hatte gleichzeitig gravierende beschäftigungspolitische Auswirkungen. Denn aufgrund der höheren Kapitalintensität sank im Zeitraum von 1960-75 die Zahl der Plantagenarbeiter um über 50000.

Mit 75,3 \% lag der ausländische Besitzanteil am Kapital des corporate sectors der Limited Companies im Agrarsektor (1970) am höchsten im Vergleich zu allen anderen Sektoren (63,3\%) (MTR - SMP: 83 und TMP: 86). 


\subsection{Kleinproduzenten}

Der letzte Bevölkerungszensus von 1970 zählte 545000 Kleinproduzentenhaushalte (Malayen: $83 \%$, Chinesen: $15 \%$ und Inder: $2 \%$ ), die ca. $60 \%$ der kultivierten Agrarfläche bewirtschafteten. Gegenüber 1957 als etwa die Hälfte der Agrarfläche auf sie entfiel, haben sie somit ihren Anteil um 10 \% oder absolut um 1,7 Mill. acres ausdehnen können, was etwa ihrer gesamten Kautschukfläche im Jahre 1957 entspricht.

Unter die Rubrik Kleinproduzenten (smallholder) fallen alle diejenigen landwirtschaftlichen Betriebe, die eine Fläche unter 100 acres bewirtschaften. Diese Klassifizierung ist jedoch wenig aussagekräftig, da zwei Drittel der Haushalte nur bis zu 5 acres verfügen und bei knapp $90 \%$ aller Haushalte die Betriebsgröße unter 10 acres liegt. Insgesamt wird nur eine Durchschnittsgröße von 5,4 acres erreicht, die bei den malayischen Kleinproduzenten mit 4,1 acres bezeichnenderweise am niedrigsten liegt.

Die-auf Betreiben der internationalen Entwicklungsagenturen Weltbank und FAO (IBRD/FAO 1975) im Jahre 1977 durchgeführten Erhebungen für einen aktuellen Agrarzensus $^{5}$ sind bislang noch nicht publiziert worden. Aus den insgesamt verfügbaren Daten, die der Chefökonom des Agrarministeriums Selvadurai in einer umfassenden Studie (Selvadurai 1978) zusammengestellt hat, läßt sich neben der Zersplitterung der bewirtschafteten Flächen eine extrem ungleiche Verteilung des Landeigentums ablesen, die in den sechziger Jahren deutlich zugenommen hat. Dies zeigt deutlich das Ansteigen des GINI-Koeffizienten der Bodenbesitzkonzentration von 0,44 im Jahre 1960 auf 0,54 im Jahre 1971 (Fu-chen Lo/Salih, K. 1977: 7). Landlosigkeit oder die Bewirtschaftung unökonomischer Betriebsgrößen - Aladin Hashim, Deputy Director von FELDA, schätzt, daß 1970 ca. 342000 Familien unter diese Klassifizierung fielen - und entsprechend niedrige Produktivitäten sind die prägenden Kennzeichen der malaysischen Agrarstruktur. $56 \%$ der Landeigentümer besitzen lediglich eine Fläche unter 3 acres, die $11 \%$ der Gesamtfläche entspricht.

Knapp $65 \%$ aller Kleinproduzenten bewirtschaften eigenes Land, während 23,5 \% Pächter sind und 11,5\% sowohl eigenes als auch zugepachtetes Land bewirtschaften. Der Eigentümeranteil liegt bei den Chinesen mit knapp drei Vierteln am höchsten, während die Inder mit 51,7 \% einen Pächteranteil ausweisen, der doppelt so hoch ist wie der Durchschnitt. Der Eigentümer-Pächter-Status liegt bei den Malayen mit 13,2\% weit über den Werten der chinesischen $(4,4 \%)$ und der indischen Kleinproduzenten $(2,5 \%)$. Der Grund dafür liegt jedoch weniger in der Erreichung einer rentablen Betriebsgröße, sondern erklärt sich vielmehr aus der extremen Landfragmentierung bei den malayischen Bauern, die u. a. auf das islamische Erbteilungsgesetz zurückzuführen ist. In vielen Fällen beinhaltet die Zupachtung von Land lediglich, daß günstiger erreichbares Land bewirtschaftet wird, während gleichzeitig eigenes weit entferntes Land selbst verpachtet wird. Diese Form der Austauschpacht wird häufig unter Verwandten im Reisanbau praktiziert. Ins- 
gesamt haben Untersuchungen in der MUDA-Region ergeben, daß Pachtverhältnisse zu ca. 2 Drittel auf Verwandtschaftsbeziehungen basieren, so daß der so gängige Terminus 'Landlord in den Reisanbaugebieten Malaysias nur begrenzt zutrifft (Ouchi et al. 1977: 53).

Insgesamt jedoch ist die Fragmentierung des Bodenbesitzes und eine stetig anwachsende Landlosigkeit, die auf eine jährliche Rate von 10000 Familien geschätzt wird, mit einer steigenden Zahl von rabsentee landlords der 'malay reservations' wirkte als Mechanismus der Förderung der Bodenbesitzkonzentration in den Händen von malayischen Bürokraten, d. h. einer sich herausbildenden städtischen Mittelklasse. Die Tatsache, daß vier Fünftel aller ıabsentee landlords` Malayen sind und in Kelantan, dem ärmsten Bundesstaat der malaysischen Föderation ihre mit Abstand höchste Anzahl ermittelt wurde, zeigt einmal mehr, daß die Inegalität nicht eine Frage der ehtnischen, sondern der Klassenzugehörigkeit ist (Evers 1978: 21).

\subsection{Land Settlement Schemes}

Die dritte Komponente der malaysischen Agrarstruktur bilden die Land Settlement Schemes. Sie entstehen im Rahmen der Neulanderschließung, dem bedeutendsten Entwicklungsprojekt des malaysischen Staates, für das bisher knapp die Hälfte aller Agrarentwicklungsausgaben alloziert wurden.

Wie schon für die Periode des SMP ist auch für den TMP die Erschließung von 1 Mill. acres Neuland projektiert worden, so daß im Jahre 1980 fast ein Viertel der kultivierten Agrarfläche auf die staatlichen Land Settlement Schemes (1970: $10 \%$ ) entfällt.

Da die Siedler der Schemes nach erfolgter Rückzahlung der Erschließungskosten Eigentumstitel für das von ihnen bewirtschaftete Land erhalten, werden sie an passender Stelle auch unter die statistische Rubrik der Kleinproduzenten subsumiert, insbesondere um einen erhöhten Besitzanteil der Malayen auszuweisen. Denn die Neulanderschließung ist gleichzeitig auch ein Instrument der Verteilungspolitik des Staates im Agrarsektor.

\section{Landpolitik und Eingriffe in die Besitzstruktur}

Wichtigstes Instrument der Landpolitik und der Einflußnahme auf die Besitzverteilung des Agrarlandes ist die Neulanderschließung. Sie bildet das Schlüsselelement der malaysischen Agrarpolitik und dient als Kompensationsstrategie gegenüber einer Land- und Agrarreform. Das Fehlen jeglicher Reformmaßnahmen zur Veränderung der Bodenbesitzstruktur und die völlige Ineffektivität von staatlichen Eingriffen zur gesetzlichen Regulierung von Pachtverhältnissen ist mit das auffälligste Charakteristikum der malaysischen Agrarpolitik. Obgleich die teils extreme Parzellierung des Bodenbesitzes, die Bewirtschaftung unrentabler Betriebsgrößen mit entsprechend niedrigen Produktivitäten und die wachsende Landlosigkeit wesentliche Ursachen der ländlichen Armut sind, ist Malaysia eines der wenigen Länder in Südostasien, das auch ansatzweise keine Landre- 
form durchgeführt hat. ${ }^{6}$ Die staatliche Regelung der Pachtverhältnisse im Reisanbau wurde 1955 erfolglos mit dem 'Padi Cultivators (Control of Rent and Security of Tenure) Act ' versucht. Auch die 1967 erfolgte Novellierung dieses völlig ineffektiven Gesetzes blieb auf dem Papier, da es aufgrund der ausbleibenden Ratifizierung durch die Mehrheit der Bundesstaaten, in deren Kompetenzbereich die Regelung des Bodenbesitzes fällt, nicht implementiert werden konnte (Fredericks/Wells 1978: 649). Eine Ursache für das Scheitern dieser gesetzlichen Regelungen ist sicherlich im hohen Grad der verwandtschaftlichen Pachtbeziehungen zu sehen.

Die staatliche Entwicklungsbehörde FELCRA hat bislang ebenfalls, obgleich als eine ihrer Aufgaben deklariert, noch keine einzige Landkonsolidierung durchgeführt. Hieran zeigt sich nur zu deutlich, daß die proklamierten Politikziele stets verbale Bekenntnisse geblieben sind, denn ihre Durchsetzung lag nicht im Interesse der herrschenden Klasse, sondern war politischen Zielen untergeordnet und wurde nicht zuletzt durch die kommunalistische Interessenbalance restringiert.

Eine aktive Verteilungspolitik wird erstmals mit dem Ziel der NEP: 'restructuring of society، verfolgt, d. h. der Umschichtung der Besitzanteile des Produktionsvermögens, die mit besonderer Priorität im Agrarsektor vorangetrieben werden soll. Aufgrund der herausragenden Rolle des Agrarsektors innerhalb der malaysischen Gesellschaft und eines gegenüber den anderen Wirtschaftssektoren überproportionalen Anteils des Auslandskapitals, der mit einem hohen Profittransfer (Bode/Müller-Debus 1978: 171) verbunden ist, sieht der Outline Perspective Plan (1970-1990) der New Economic Policy mit einer jährlichen negativen Veränderungsrate von - 2,7 \% auch einen absolut sinkenden ausländischen Besitzanteil vor (MTR-SMP: 86,7), während in den übrigen Sektoren die Umverteilung nur über das Wachstum erfolgen soll. Insgesamt ist das Verteilungsziel zwar nicht sektoral spezifiziert worden, sondern lediglich für den Sektor der natürlichen Ressourcen wurde ein inländischer, malaysischer Besitzanteil von $70 \%$ projektiert. Dagegen werden im exportorientierten, hochtechnologischen Industriesektor durchaus höhere ausländische Besitzanteile akzeptiert (TMP: 278). Sichtbares Resultat dieser Malayisierungspolitik, die mit marktwirtschaftlichen Mitteln über den börsenmäßigen Ankauf von Aktienanteilen praktiziert wird, ist ein sukzessiver Rückgang des Flächenbesitzes der ausländisch kontrollierten Plantagengesellschaften, der bei Kautschuk von 1967 bis 1975: 13,3 \% und bei Olpalme von 1969 bis 1975: 28,6 \% betrug. Hauptakteur dieser Malayisierungspolitik ist der Staatskonzern Pernas mit seinen Untergesellschaften, der bereits beträchtliche Kapitalanteile erworben hat ${ }^{7}$ und seinen Einfluß auf den britischen Konzern Sime Darby und seine Interessen soweit durchsetzen konnte, daß sein Sitz nach Kuala Lumpur verlegt wurde. ${ }^{8}$

6 Die dringende Forderung nach Durchführung einer Landreform wurde einmal mehr während der 'Ninth Great Economic Debate on National Land Problems - A Claim for Reformation von Wissenschaftlern und dem Führer der Oppositionspartei, PI, Datuk Asri Muda gestellt. Vgl. New Straits Times (Kuala Lumpur) v. 23. 9.1978.

7 Diese Kapitalbeteiligungen der Staatskonzerne sind später dann auf die staatliche Investment-Treuhandgesellschaft Permodalan Nasional Berhad (PNB) übertragen worden.

8 Zum Aufsichtsratsvorsitzenden wurde der ehemalige Finanzminister Tun Tan Siew Sin gewählt 
Sime Darby ist damit Malaysias erster eigener Multinationaler Konzern geworden, ${ }^{9}$ der seinerseits weitere Gesellschaften, wie z. B. Guthrie und Dunlop unter seine Kontrolle zu bringen versucht. Neben der Ưbernahme von Plantagenfläche durch Staatskonzerne, die statistisch als malayischer Besitz ausgewiesen wird, muß der Umschichtungsprozeß im privaten Sektor eindeutig zugunsten der chinesischen Bourgeoisie erf olgt sein, denn nach Angaben des Ministers für Land and Regional Development Tan Sri Kadir beläuft sich der private malayische Besitzanteil auf minimale $1 \%$ bei der Kautschuk- und $5 \%$ bei der Olpalmfläche. ${ }^{10}$

\section{Instrumente der Agrarpolitik}

Das zentrale Instrument staatlicher Politik im exportorientierten Segment des Agrarsektors ist die Erschließung von Neuland, die sowohl die Exportproduktion steigert als auch Arbeitsplätze schafft und gleichzeitig die Diversifizierung der Produktstruktur ermöglicht. Daneben bildet die Modernisierung des veralteten und daher wenig produktiven Baumbestandes der Kautschukbauern ein wichtiges Instrument zur Förderung der in-situr Entwicklung im Exportsektor. Ein wesentliches Element dabei liegt in der technologischen Entwicklung von Hochertragsbäumen. Das Ziel der Importsubstitution von Reis im Nahrungsmittelsektor versucht der malaysische Staat mit Hilfe von großangelegten regionalen Bewässerungsprojekten einschließlich der Einführung der 'Green Revolution' zu erreichen.

\subsection{Neulanderschließung}

Der postkoloniale Staat übernahm die Neulanderschließung, d. h. die Inwertsetzung der Ressource Land in eigene Regie und reduzierte systematisch die Möglichkeit für die Plantagen ihre Fläche über die Erschließung von Neuland auszudehnen. Hauptakteur der Neulanderschließung ist die 1956 gegründete Bundesbehörde Federal Land Development Authority (FELDA). Daneben verfügen die Bundesstaaten über eigene Siedlungsgesellschaften, von denen die Kelantan State Land Development Board (KSLDB) von besonderer Bedeutung ist. Bedingt durch den politischen Konflikt zwischen der Alliance - Bundesregierung und der PI, die Kelantan von 1959-78 regierte, führte die KSLDB ein zu FELDA grundsätzlich alternatives Konzept durch, das wesentlich auf der Eigenarbeit und der Partizipation der Siedler basiert und zusätzlich die Selbstversorgung mit Nahrungsmitteln - außer Reis - einschließt.

Dagegen ist FELDA's Konzept äußerst kapitalintensiv - die Kosten einer Siedlerstelle entsprechen fast denjenigen eines industriellen Arbeitsplatzes -, denn die Siedler ziehen

9 Vgl. Far Eastern Economic Review/FEER v. 13. 1. 1978 und v. 7. 9. 1979. Auch über den größten Zinnkonzern der Welt 'London Tin hat Pernas mit Hilfe seiner Tochtergesellschaft New Tradewinds die Kontrolle übernommen; vgl. FEER v. 1. 4. 1977.

10 Vgl. New Straits Times v. 28. 3. 1978. 
in ein von Privatfirmen erschlossenes und voll funktionsfähiges Scheme ein. Zudem hat FELDA im Gegensatz zu KSLDB eine extrem bürokratische Administration der Schemes entwickelt. Während das Personal-Siedler-Verhältnis im KSLDB-Scheme bei 1:80 liegt, beträgt es bei FELDA mit 1:7 mehr als das Zehnfache (Rokiah Talib 1978). Mit einem Anteil von $46 \%$ ist knapp die Hälfte der gesamten Agrarentwicklungsausgaben auf die Neulanderschließung verwendet worden, die damit eindeutige Priorität innerhalb der malaysischen Agrarpolitik besitzt. Sie war zudem politisch ohne Schwierigkeiten durchsetzbar, denn die chinesische Bourgeoisie profitierte von den Auftragsarbeiten, das Auslandskapital von der Exportproduktion und die malayische Elite zog den politischen Nutzen daraus.

Von 1956-78 hat allein FELDA ca. 1 Mill. acres erschlossen und über 40000 von insgesamt 70000 Familien angesiedelt. Für die Planperiode des TMP ist ebenfalls die Erschließung von 1 Mill. acres projektiert worden, wobei der private Sektor einen Anteil von 26 \% (TMP: 302) übernehmen soll. Im Jahre 1976 hatte FELDA 35 \% seiner Fläche mit Kautschuk und $63 \%$ mit Olpalme bepflanzt und geringe Anteile mit Zuckerrohr und Kakao. FELDA verfügt über $7 \%$ der Kautschuk-, aber über ein Drittel der gesamten Olpalmfläche West-Malaysias und hat damit wesentlich dazu beigetragen, daß Malaysia mit einem Anteil von 40 \% zum der Welt größten Exporteur von Palmöl aufgestiegen ist. Angesichts einer drohenden Angebotslücke von Naturkautschuk ${ }^{11}$ werden zur Zeit jedoch wieder mehr Kautschukbäume im Rahmen einer 'Dynamic Rubber Production Policy ${ }^{12}$ gepflanzt.

Konstitutives Element des FELDA-Konzepts ist die Ubertragung von Eigentumstiteln an die Siedler nach erfolgter Rückzahlung der Erschließungskosten, denn lediglich die administrativen Kosten werden staatlich subventioniert. Somit ist die kapitalintensive Landerschließung mit einer gravierenden finanziellen Belastung der Siedler für einen Zeitraum von mindestens 20 Jahren verbunden. Das projektierte Einkommensziel von 300-350 M\$ ist daher auch erst seit Mitte der siebziger Jahre als Folge einer äußerst günstigen Entwicklung der Weltmarktpreise erreicht worden. Auffälliges Charakteristikum des FELDA-Konzepts ist die extreme Einkommensungleichheit zwischen Kautschuk- und Olpalmbauern, die zwar durch die unterschiedliche Preishöhe auf dem Weltmarkt verursacht, aber auch nicht intern ausgeglichen wird. So lag das durchschnittliche monatliche Nettoeinkommen einer Siedlerfamilie 1974 in einem Kautschukscheme bei $250 \mathrm{M} \$$ und einem Olpalmscheme dreimal so hoch bei $819 \mathrm{Ms}$.

Mit 10-14 acres verfügt der FELDA-Siedler über eine Fläche, die mehr als doppelt so groß ist wie der Landesdurchschnitt. FELDA kreiert somit eine neue, kleine Schicht von Mittelklassebauern. In den Genuß dieser privilegierten Position kommen fast ausschließlich Malayen, die die Siedlerstelle zumeist als Bonus für politisches Wohlverhalten gegenüber der UMNO erhalten (Robertson 1975), d. h. FELDA wird wesentlich auch als Instrument der Verteilungspolitik zugunsten der Malayen funktionalisiert, was

11 Grilli et al. (1978) prognostizieren das Angebotsdefizit für 1990 auf ca. 500000 to.

12 Natural Rubber News, Sept. 1980, p. 10. 
eindeutig im Widerspruch zum erklärten Ziel der NEP steht, die Armut unabhängig von der jeweiligen ethnischen Zugehörigkeit zu bekämpfen.

\subsection{Modernisierung der Kautschukproduktion}

Die Gründe für die bereits zu Beginn der fünf ziger Jahre einsetzende Modernisierungspolitik im Kautschuksektor lagen sowohl in internen Angebots- als auch in externen Nachfragefaktoren. Letztere wurden wesentlich von der Substitutionskonkurrenz mit Synthesekautschuk (Jägeler 1976) bestimmt. Daher war es nicht nur notwendig, die Konkurrenzposition von Naturkautschuk zu stärken, sondern insbesondere die Tatsache, daß im Jahre 1952 knapp $60 \%$ des Baumbestandes der Kleinproduzenten, die immerhin $44 \%$ der Kautschukfläche bewirtschafteten, das ökonomische Lebensalter bereits überschritten hatte, erforderte eine dringende Modernisierung, um ein ständiges Sinken der Produktivität, der Produktion und damit der Exporte einschließlich der Staatseinnahmen aus der Exportsteuer zu verhindern. Die Modernisierung lag somit auch in einem manifesten Eigeninteresse des Staates. Wesentliches Instrument des $/$ Replanting-Programms` bildete die Errichtung zweier getrennt arbeitender Replanting Fonds jeweils für die Plantagen und die Kleinproduzenten, dessen Verwaltung 1973 die Rubber Industry Smallholder Development Authority (RISDA) vom Rubber Industry Replanting Board übernahm. Finanziert wurden die Fonds, die auf Drängen der Plantagenlobby gespalten wurden, um einen Einkommenstransfer zu verhindern, über eine zusätzliche Exportsteuer (Replanting Cess). Während die Plantagen nur ein Zwangssparen durchführten, denn sie erhielten exakt den von ihnen eingezahlten Betrag zurückgezahlt, war die Arbeitsweise des Fonds für die Kleinproduzenten mit einer beträchtlichen Umverteilung verbunden. Bis 1976 hatten die Kleinproduzenten $68 \%$ ihrer Fläche mit Hilfe der Replanting-Prämien modernisiert, die von 1952 bis 1979 kontinuierlich von 400 auf $1300 \mathrm{M} \$$ pro acre erhöht worden sind.

Obwohl keine nach Farmgröße spezifizierten Daten verfügbar sind, liegt doch die Vermutung nahe, daß nur die Bauern mit einer Mindestfläche von 5 acres das Replanting durchgeführt haben, denn die noch nicht modernisierte Fläche von 732000 acres entspricht fast genau dem Flächenanteil, der auf die Bauern mit einer Farmgröße bis zu 5 acres (758 000 acres) entfällt. Insofern hat also ein indirekter Einkommenstransfer von den kleinen Betrieben, die nur eingezahlt haben, auf die größeren stattgefunden. Insgesamt haben die Kleinbauern einen absolut höheren Betrag für den Fonds aufgebracht als sie später in der Form der Replanting-Prämie zurückerhielten. Berechnungen haben zudem ergeben, daß eine Anlage als Sparkapital den Bauern einen weitaus höheren Ertrag gebracht hätte, den insbesondere auch die Kleinstbauern hätten realisieren können (Khoo Khay Jin 1978). ${ }^{13}$

Neben dem regressiven Verteilungsmechanismus impliziert dies außerdem einen gewis-

13 Barlow (1978: 267) kommt ebenfalls zu dem Ergebnis, daß über die Replanting Cess ein übermäßig hoher Einkommensanteil von den Bauern absorbiert wurde. 
sen Dekapitalisierungseffekt, der jedoch in einem weitaus höheren Maße durch die extrem hohe Exportsteuerbelastung erzielt wird, der die Kautschukbauern verglichen mit städtischen Einkommensbeziehern übermäßig belastet.

Trotz dieser erheblichen Kaufkraftabschöpfung durch den Staat und der instabilen Preisentwicklung auf dem Weltmarkt hat jedoch auf über zwei Drittel der Kleinproduzentenfläche Produktivkraftentwicklung stattgefunden. Da das Replanting auf der Basis der bestehenden Landbesitzverteilung stattfand, war es mit einem negativen Verteilungseffekt verbunden, der zu einer tendenziellen Polarisierung in akkumulations- und nichtakkumulationsfähige Bauern geführt hat, die wenig produktive Kleinstflächen bewirtschafteten und weiterhin die Gruppe der Armutsbauern bilden. Denn letztlich konnten nur diejenigen Bauern die seit 1975 gestiegenen Kautschukweltmarktpreise in spürbare Realeinkommenssteigerungen umsetzen, die zuvor entsprechende Produktivitätsfortschritte realisiert hatten.

Wesentlicher Bestandteil der Modernisierungspolitik ist die Arbeit des staatlichen Rubber Research Institutes (RRI) in Kuala Lumpur, das sowohl von den Plantagen als auch den Kleinbauern über eine Forschungssteuer (research-cess) finanziert wird. Das RRI versorgt die Bauern mit Hochleistungsbäumen, die damit ihre Jahresproduktion pro acre verdoppeln konnten. Dagegen lag die Steigerungsrate der Plantagen mit $265 \%$ weitaus höher. Insgesamt erreichen die Kleinbauern zwei Drittel der Plantagenproduktivität. Hierbei handelt es sich jedoch nur um einen Durchschnittswert, der zudem die Produktion der Land Settlement Schemes mit einschließt, so daß die Produktivität der Kleinbauern sicher weit niedriger einzuschätzen ist. Im internationalen Vergleich der Produktivitätsentwicklung hat sich Malaysia eine unangefochtene Spitzenstellung erobert. So ist dort die Flächenproduktivität doppelt so hoch wie in Sri Lanka und beträgt das Vierfache derjenigen Thailands. ${ }^{14}$ Im Zeitraum von 1957-77 ist die Produktion von 600000 auf über 1,5 Mill. Tonnen gestiegen, die zu $58 \%$ aus dem Kleinbauernsektor (1957: $42 \%$ ) stammen. Insgesamt ist Malaysias Anteil an der Weltproduktion um $10 \%$ auf $42 \%$ angewachsen.

Die Beschäftigungsproduktivität hat sich nach Berechnungen von v. Rabenau (1977: 28,9 ) im Zeitraum von 1957-76 bei den Plantagen verdreifacht, bei den Kleinproduzenten jedoch nur verdoppelt. Ein gravierender Unterschied zeigt sich bei der Entwicklung der Relation der Anbaufläche pro Beschäftigten. Diese ist bei den Plantagen aufgrund kapitalintensiver Bearbeitungsmethoden um 0,8 acres gestiegen, während die Kleinproduzenten eine Verschlechterung um einen acre hinnehmen mußten, obgleich ihre Fläche absolut um 1 Mill. acres expandierte. Somit konnte das hohe Bevölkerungswachstum nicht aufgefangen werden, sondern die Verschlechterung verdeutlicht einmal mehr, daß die ständig steigende Landknappheit eine der wesentlichen Ursachen der Armut der Kautschukbauern bildet. Der Pächteranteil ist von $10 \%$ im Jahre 1960 auf zur Zeit ca. $40 \%$ angestiegen. Während die durchschnittliche Betriebsgröße bei 5,5 acres liegt, ist nach den Berechnungen der Weltbank/FAO-Teams eine Mindestfläche von 7,5 acres 
zur Erwirtschaftung eines Basiseinkommens zur Befriedigung der Grundbedürfnisse für eine Kleinbauernfamilie notwendig (IBRD/FAO 1975: 77).

\subsection{Preisstabilisierungspolitik}

Trotz erheblicher Preisfluktuationen hat der Staat erst ein einziges Mal preisstabilierend interveniert. Als nach dem dramatischen Sturz des Kautschukpreises von 265 cts. auf 97 cts. im November 1974 die Bauern von Baling Hungermärsche organisierten, da gleichzeitig die Nahrungsmittelpreise um 50-200\% in die Höhe geschnellt waren und auch die Studenten in Kuala Lumpur zur Unterstützung der Forderungen der Bauern nach Hilfe des Staates auf die Straße gingen, reagierte der Staat mit einem Crash-Programm. Durch Produktionsdrosselungen bei den Plantagen und den Aufkauf von bereits produziertem Kautschuk von den Kleinproduzenten wurde das Angebot um 190000 Tonnen für ein Jahr reduziert. Untersuchungen von Lim Sow Ching (1976: 24) zufolge ist dadurch ein preisstabilisierender Effekt eingetreten. Aber nicht so sehr ökonomische als vielmehr politische Gründe waren für die Implementation dieses Programms ausschlaggebend.

Ein erster Schritt zur außenwirtschaftlichen Stabilisierung des Kautschukweltmarktpreises war die Gründung und Malaysias Beitritt zur Association of Natural Rubber Producing Countries (ANRPC) mit Sitz in Kuala Lumpur. Als effektiv organisierte und mit einer Stimme auftretende Produzentenvereinigung war sie die treibende Kraft bei den Verhandlungen des Internationalen Kautschukabkommens, das im Oktober 1979 als erstes Rohstoffabkommen im Rahmen des integrierten Rohstoffprogramms der UNCTAD abgeschlossen werden konnte (Kasch 1981).

\subsection{Importsubstitution der Reisproduktion}

Die Importsubstitutionspolitik, die den extrem hohen Grad der Importabhängigkeit (1957 : $46 \%$ ) als strukturelle Folge der Exportspezialisierung der malaysischen Okonomie vollständig abbauen sollte, wurde wesentlich durch folgende ökonomische und politische Faktoren beeinflußt. Langfristig war die Selbstversorgung mit Reis, dem Hauptnahrungsmittel, ein dringendes Erfordernis, um die Bevölkerung vor Nahrungsmittelkrisen und entsprechend drastischen Preissteigerungen (1973/74) zu schützen und natürlich auch um Devisen einzusparen. Dem Selbstversorgungsmotiv widersprach jedoch das ökonomische Kostenkalkül, da die Reisproduktion in Malaysia eindeutig mit komparativen Kostennachteilen gegenüber dem Importreis verbunden war. Ein steigender Grad der Selbstversorgung implizierte damit zugleich auch steigende Konsumentenpreise sowohl für die städtischen Bewohner als auch für die Plantagenarbeiter und die spezialisierten Kautschukproduzenten, d. h. die Reproduktionskosten eines Großteils insbesondere auch der ländlichen Bevölkerung würden erheblich ansteigen.

Diesen Konflikt löste der Staat mit einer gemischten Strategie von Preis- und Vermarktungspolitik. Den Bauern wurde ein Minimumpreis offiziell garantiert und der Staat ver- 
band die Vergabe von Importlizenzen mit der Auflage, eine Quote der staatlich aufgekauften Reismenge zu übernehmen, so daß ein Mischpreis zwischen internem und Importpreis entstand und somit z. T. die Kosten der Importsubstitutionspolitik für die Konsumenten reduziert wurden, insbesondere auch gegenüber einer Schutzzollpolitik. Letztlich aber war eine aktive Wachstumspolitik im Reissektor von der Notwendigkeit bestimmt, die größtenteils noch auf Subsistenzniveau produzierenden Reisbauern in den Markt zu integrieren und die Kommerzialisierung ihrer Produktion voranzutreiben. Im Jahre 1970 wurde erst etwa die Hälfte der gesamten Reisproduktion überhaupt vermarktet und bei knapp der Hälfte der Haushalte wurde noch nicht einmal der Eigenverbrauch gedeckt (Goldman 1975 : 286).

Seinen besonderen Akzent erhält die Politik des Staates im Reissektor aufgrund des ethnischen Faktors. Die Reisproduzenten sind fast ausschließlich (97\%) Malayen. Sie weisen mit knapp $90 \%$ der Haushalte den höchsten Armutsanteil aller Beschäftigungsgruppen auf, so daß die gängige Identifizierung von ländlicher Armut mit malayischer Armut hier zutreffend ist. Somit ist Reis in Malaysia in vielfacher Hinsicht eine eminent 'political crop'.

Hauptinstrument der Importsubstitutionspolitik im Reissektor, die insbesondere im Rahmen des ersten Malaysia Plans (1966-70) vorangetrieben wurde, sind großflächige Bewässerungsprojekte in den traditionellen Reisanbaugebieten, den sogenannten rice bowls‘. Das größte ist das zu $60 \%$ mit Hilfe von Weltbankkrediten finanzierte MUDAProjekt in Kedah/Perlis mit 260000 acres, das allein schon über ein Viertel der gesamten Reisanbaufläche West-Malaysias (944 000 acres) umfaßt (Jegathesan 1977). Weitere Projekte sind: KEMUBU in Kelantan (80 000 acres), Krian in Perak (67 000 acres) und Tanjong Karang in Selangor (46 000 acres). Der Zugang der Reisbauern zur staatlichen Modernisierungspolitik war damit von vornherein durch den Standort bestimmt.

Bis 1975 konnten drei Viertel der Reisanbaufläche mit künstlicher Bewässerung versorgt werden und der Anteil der Fläche mit einer jährlichen Doppelernte ist von unter einem Prozent im Jahre 1957 auf knapp $60 \%$ gesteigert worden.

Die Verdoppelung der Reisproduktion von 1960 bis Mitte der siebziger Jahre, wo sie ihr Maximum erreichte und den Grad der Selbstversorgung auf $90 \%$ anhob, ist hauptsächlich auf diese Flächenexpansion qua Einführung einer jährlichen Zweiternte zurückzuführen und nur geringfügig auf eine gestiegene Produktivität. Seit 1975 ist die Reisproduktion u. a. aufgrund schlechter Wetterbedingungen wieder absolut gesunken. Scheinbar ist sie z. Zt. an ihre Wachstumsgrenze gestoßen.

Trotz Einführung von High Yielding Varieties seit 1965 weisen die Erträge nur äußerst bescheidene Wachstumsraten auf. Von 1963-1975 stieg der Ertrag pro acres in der 'mainseason' minimal von 411 auf 444 Gantangs und in der soffseason' um immerhin 75 auf 511 Gantangs (Selvadurai 1978:78). Dennoch erwirtschaften die malaysischen Reisbauern die höchste Flächenerträge in Südostasien.

Die Modernisierung und Kommerzialisierung der Reisproduktion ist in der MUDA-Region mit seinen 60000 Reisbauernfamilien am weitesten vorangetrieben worden. Bis heute konnte dort die Produktion seit Inangriffnahme des Projekts um das Dreieinhalb- 
fache gesteigert werden, die damit knapp die Hälfte des malaysischen Reisverbrauchs deckt (1965: $26 \%$ ). Die Kommerzialisierung der Produktion hat sich mit einem Anteil der Marktproduzenten von $90 \%$ gegenüber den fünfziger Jahren, als weniger als $40 \%$ der Reisbauern für den Markt produzierten (Koninck, De 1979 : 282,3), nahezu vollständig durchgesetzt.

\subsection{Die Einkommensentwicklung der Reisbauern}

Die Tatsache, daß die Reisbauern mit knapp $90 \%$ den höchsten Armutsanteil aufweisen, ist ein deutliches Indiz dafür, daß die Politik des Guaranteed Minimum Price (GMP) zur Stabilisierung der Einkommen der Reisbauern auf einem ausreichenden Niveau zumindest bis 1970 fehlgeschlagen ist. Der GMP, der von 1963-75, also über 10 Jahre konstant $16 \mathrm{M} \$$ pro picul Reis betrug, und der erst infolge der weltweiten Nahrungsmittelkrise sukzessive auf zur Zeit ca. $30 \mathrm{M} \$$ erhöht wurde, konnte aufgrund der institutionellen Struktur des Vermarktungssystems kaum durchgesetzt werden. Immer noch sind die malayischen Bauern der Preiswillkür der zumeist chinesischen Händler ausgeliefert. Die staatliche Reisbehörde National Padi and Rice Authority konnte sich erst einen Marktanteil von knapp einem Viertel sichern, der in der MUDA-Region am höchsten liegt und einmal mehr die privilegierte Position der dort lebenden Reisbauern unterstreicht. Lediglich im Tanjong-Karang-Projekt existiert ein staatliches Vermarktungsmonopol.

Im MUDA-Projekt, dem 'show-piecer der malaysischen Agrarpolitik kristallisiert sich ein zwar ungleicher, aber insgesamt doch positiver Trend der Einkommensentwicklung heraus. Nach Berechnungen von Jegathesan sind die durchschnittlichen Realeinkommen der MUDA-Bauern von 1966-75 um 141 \% gestiegen. Jedoch wurden diese reale Steigerung durch die drastische Erhöhung der Produktionskosten, deren Index sich von 1970 : 100 bis zum Jahre $1977: 295$ fast verdreifachte, weitgehend wieder aufgezehrt und auch die Pachtraten haben sich von 1966-75 verdoppelt (Jegathesan 1977 : 38,61). Bell/Hazell ermittelten für die Periode von 1964-1974 ein durchschnittliches jährliches Wachstum der Realeinkommen aller Haushalte in der MUDA-Region von 7,5\% (Bell/ Hazell 1980 : 82), d. h. nicht nur Haushalte mit einem Landüberschuß sondern auch landlose Reisarbeiterhaushalte haben somit über steigende Reallöhne an diesem Einkommenszuwachs partizipiert. Insgesamt weist die Einkommensentwicklung zwei signifikante Tendenzen auf. Zum einen zeigt sich eine erhebliche einkommensdifferenzierende Wirkung der staatlichen Modernisierungspolitik, die einen sichtbaren Prozeß der sozialen Differenzierung und Klassenbildung im Reissektor inganggesetzt hat. Denn die ungleiche Landverteilung führte nicht nur zu einer ungleichen Verteilung der Einkommenszuwächse, sondern sie versetzte zudem diejenigen Bauern, die Ưberschüsse akkumulieren konnten, in die Lage, mit Hilfe eines steigenden Mechanisierungsgrades ihre Flächen auszudehnen und vorher verpachtetes Land wieder selbst zu bewirtschaften. Eine steigende Bodenbesitzkonzentration und die Freisetzung von ca. 7000 Pächtern, die immerhin mehr als $10 \%$ der Bauernfamilien in der MUDA-Region ausmachen, sind die 
negativen Folgen einer lediglich auf Kapitalinvestitionen beruhenden Modernisierungspolitik, die in keiner Weise von einer Bodenreform oder Pachtgesetzen flankiert wird. Die Tatsache, daß dieser Prozeß der Trennung der Pächter vom Produktionsfaktor Boden bisher zumindest nicht zu deutlich sichtbaren Marginalisierungstendenzen geführt hat, ist einerseits auf die Verwandtschaftsbasis der Pachtbeziehungen zurückzuführen und andererseits darauf, daß ein Teil der freigesetzten Pächter Beschäftigung in der nahegelegenen Freien Produktionszone von Penang gefunden hat.

Zum anderen weist die Einkommensentwicklung mit der Konzentration der Zuwächse auf die Nichtagrarhaushalte in der MUDA-Region (Bell/Hazell 1980: 82) einen deutlichen surban-biası auf. Nach Berechnungen der Weltbank fließt von dem mit der Reisproduktion erwirtschafteten Úberschuß von 250 Mill. M \$ jährlich ein Nettobetrag von über 100 Mill. M\$ wieder aus der MUDA-Region ab. ${ }^{15}$ Denn die Reisbauern wurden nicht nur als Produzenten, sondern auch als Konsumenten in den Markt integriert. Ihre Nachfrage nach Konsumgütern und speziell Produktionsmitteln, die nicht in der MUDA-Region produziert werden, löst einen kontinuierlichen Ressourcentransfer in den städtisch-industriellen Sektor aus. Dieser strukturelle Mechanismus verhindert die Nutzung der durch die gestiegene Reisproduktion erzielten Einkommenszuwächse zum Aufbau eines eigenständigen, regionalen Wirtschaftskreislaufes, der auf der funktionalen Integration von Agrar- und Industriesektor basiert. Statt dessen führt das Wachstum zu einer weiteren Verschärfung der bereits existenten regionalen, sektoralen und nicht zuletzt der sozialen Disparitäten. Erreichte Kedah im Jahre 1963 noch ein Pro-KopfEinkommen in Höhe von $87 \%$ des Landesdurchschnitts, so ist dieser Wert infolge des äußerst ungleichgewichtigen Wachstumsprozesses auf $54 \%$ im Jahre 1978 gesunken, trotz der massiven staatlichen Investitionen in dieser Region.

\section{Wachstum und Verteilung: Widersprüche der Agrarpolitik}

Von der Outputseite betrachtet, bietet der malaysische Agrarsektor ein recht prosperierendes Bild, das die Agrarpolitik des malaysischen Staates als offensichtlich recht erfolgreich ausweist. Dennoch lagen die Wachstumsraten der Agrarproduktion mit ca. $4 \%$ weit unter den gesamtwirtschaftlichen von 6-8\%, so daß sich der Beitrag des Agrarsektors zur Erwirtschaftung des BSP von 1957: 43,3\% auf 1978: 27,7 \% erheblich reduzierte. Dies ist jedoch weniger auf eine relative Vernachlässigung des Agrarsektors zurückzuführen als vielmehr auf überproportional hohe Wachstumsraten der verarbeitenden Industrie (1971-75: 10,9 \%). Bezogen auf die Exporte und die Beschäftigung fiel der relative Rückgang jedoch weitaus bescheidener aus.

Weiterhin besteht über die Hälfte der Exporte (1978: 52 \%; 1957: $66 \%$ ) aus Agrarprodukten und in knapp 20 Jahren sank der Beschäftigungsanteil lediglich um $10 \%$ (1975: 46,2\%). Malaysias Wirtschafts- und damit auch Gesellschaftsstruktur besitzt auch heute noch einen dominant agrarischen Charakter. Der sozioökonomische Strukturwandel 
vollzog sich in einem recht langsamen Tempo insbesondere angesichts eines verglichen mit anderen asiatischen Ländern weitaus höheren Pro-Kopf-Einkommens. Úber zwei Drittel der Bevölkerung (68 \%) leben noch in ruralen Gebieten. Das im Vergleich mit anderen Peripherieländern äußerst niedrige Ausmaß der rural-urbanen Migration liegt zum einen darin begründet, daß im Agrarsektor über 265000 zusätzliche Arbeitsplätze geschaffen wurden, was etwa der Gesamtbeschäftigung der verarbeitenden Industrie im Jahre 1970 entsprach. Zum anderen bildete der Sektor der verarbeitenden Industrie noch keinen bedeutenden Pullfaktor der Migration, da er im gleichen Zeitraum seinen Beschäftigungsanteil nur um etwas über $3 \%$ steigern konnte. In den letzten Jahren ist jedoch eine zunehmende Tendenz der Landflucht insbesondere von Jugendlichen zu beobachten, so daß in einigen Regionen, die im Einzugsbereich industrieller Zentren liegen (z. B. Penang und Singapur) im Agrarsektor und insbesondere auf den Plantagen bereits Arbeitskräfteknappheit herrscht.

Absolut hat die Agrarproduktion eine recht beachtliche Höhe erreicht. Malaysia ist nicht nur der Welt größter Produzent und Exporteur von Kautschuk, sondern auch von Palmöl. In der beachtlich kurzen Zeitspanne von 1964-77 verzwölffachte Malaysia seine Palmölproduktion und erreichte damit einen Anteil von $40 \%$ an der Weltproduktion. Dieses Produktionswachstum beinhaltete zudem eine beträchtliche Diversifizierung der Agrarexporte. Erwirtschaftete allein Kautschuk im Jahre 1960 bereits $55 \%$ der gesamten Exporterlöse, so entfällt zur Zeit ein geringerer Anteil (52\%) - bei gleichzeitiger wertmäßiger Verdreifachung - auf eine Palette von Agrarprodukten: Kautschuk, Palmöl, Hölzer und Pfeffer, der in Ostmalaysia produziert wird. Damit rangiert Malaysia auf Platz zwei hinter Brasilien auf der Liste der Welt größten Rohstoffexportländer außer Erdöl. Trotz dieser erfolgreichen Stärkung der Außenhandelsposition hat sich Malaysias Stellung und Kompetenz innerhalb der internationalen Arbeitsteilung als weltmarktabhängiger Rohstoffproduzent nicht wesentlich verändert, denn eine Dynamisierung der komparativen Vorteile über einen steigenden Weiterverarbeitungsgrad der eigenen Rohstoffe hat bisher nicht in einem nennenswerten Ausmaß stattgefunden. Noch nicht einmal $3 \%$ der Kautschukproduktion werden im Lande weiterverarbeitet. Im Nahrungsmittelsektor, d. h. speziell bei Reis hat die Importsubstitutionspolitik die Reisproduktion verdoppelt und damit den Grad der Selbstversorgung von 1957: $54 \%$ auf derzeit ca. $90 \%$ erhöht. Eine Wachstumsrate der Nahrungsmittelproduktion, die mit 4,8 \% (1970-77) über dem Bevölkerungswachstum lag, führte zu einer kontinuierlichen Verbesserung der internen Nahrungsmittelversorgung. Der Pro-Kopf-Index der Nahrungsmittelproduktion stieg von 1961/63: 100 auf 1974/76: 144 und erreichte damit die höchste Steigerungsrate in Südostasien (Griffin 1979: 373).

Ein quantitatives Wachstum einschließlich einer gestiegenen Produktivität der Agrarproduktion, die Diversifizierung der Exportstruktur, sowie ein positiver Beitrag zur Beschäftigung und zur internen Kapitalbildung können als beachtliche Erfolge der Agrarpolitik des malaysischen Staates verbucht werden, der damit einen entscheidenden Beitrag zur Produktivkraftentfaltung des Agrarsektors geleistet hat. Verläßt man aber die Ebene der Makro- und der Durchschnittsdaten und stellt die Frage nach dem Cui bono? 
des Wachstums, so offenbart sich das Bild einer extremen Ungleichverteilung der realen Nutzeffekte. Der Wachstumsprozeß der malaysischen Okonomie hatte auch in den siebziger Jahren zu einer Verschlechterung der personellen Einkommensverteilung, sowie der Ausweitung der sektoralen und der regionalen Disparitäten geführt. War Malaysia bereits im Zeitraum von 1957-70 mit einem Anstieg des GINI-Koeffizienten von 0,412 auf 0,510 in die Ländergruppe mit der höchsten Einkommenskonzentration aufgerückt, so läßt sich aus den verfügbaren Daten ${ }^{16}$ ablesen, daß sich dieser Prozeß weiter verstärkt hat (Snodgrass 1980: Kap. 4). Angesichts der beträchtlichen Zunahme der Einkommensinegalitäten müssen die von der offiziellen Statistik ausgewiesenen Realeinkommenssteigerungen vorsichtig interpretiert werden. Denn während in der ersten Phase des malaysischen Entwicklungsweges über $60 \%$ der ländlichen Bevölkerung erhebliche Realeinkommensrückgänge hinnehmen mußten, sind nach den Angaben des Mid-Term-Review des TMP im Zeitraum von 1970-76 auch die Einkommen der untersten $40 \%$ der ländlichen Einkommensbezieher real um insgesamt $25 \%$ gestiegen. Entsprechend weist er auch die erfolgreiche Reduktion des Anteils der Armutshaushalte im Agrarsektor von 68,3\% (1970) auf 54,6\% im Jahre 1978 und absolut um 70000 Haushalte aus (MTRTMP: 27,8). Gegenüber diesem insgesamt gesehen recht positiven Trend der Einkommensentwicklung hat die obige Analyse gezeigt, daß innerhalb der Bauernschaft gerade auch als Folge der staatlichen Modernisierungspolitik aufgrund ihres technokratischen Charakters und ihrer Konzentration auf kapitalintensive Projekte sowie der einkommensdifferenzierenden Wirkung und den negativen Verteilungseffekten ein deutlicher Stratifizierungsprozeß eingesetzt hat. Während sich auf der einen Seite ein Mittelklassebauer herausbildet, der bewußt in den Land Settlement Schemes kreiert wird, vertieft sich auf der anderen Seite die Armutsposition von nicht akkumulationsfähigen Bauern, die strukturell von der Modernisierungspolitik des Staates ausgeschlossen sind.

\section{Staat und Entwicklung}

Malaysias Entwicklungsweg ist ein Beispiel dafür, daß hochgradige Weltmarktabhängigkeit nicht zwangsläufig zur fatalen Dialektik von Wirtschaftswachstum und Ausbreitung von Massenelend führen muß. Auf der Basis einer reichen Ressourcenausstattung und in letzter Zeit auch günstiger außenwirtschaftlicher Faktoren hat der postkoloniale Staat von Anfang an - u. a. auch in bruchloser Fortsetzung kolonialer Politik - getragen von der Interessenallianz der malayischen Herrschaftselite und des Auslandskapitals eine aktive Agrarpolitik betrieben. Sie hat damit nicht nur einen wichtigen Beitrag zum Wachstum der Agrarproduktion geleistet, sondern auch insgesamt zur Produktivkraftentwicklung des Agrarsektors, die bei den Bauern auch zu realen Einkommenszuwächsen geführt hat.

16 Die Tatsache, daß die Regierung die Ergebnisse von 3 Stichprobenerhebungen, die in den siebziger Jahren durchgeführt worden sind, u. a. ein Agrarzensus, nicht vollständig, sondern lediglich selektiv, wie z. B. im Mid-Term-Review des Third Malaysia Plan, veröffentlicht hat, unterstützt diese Vermutung. 
Die Wahrnehmung der Modernisierungsfunktion, die sich im Rahmen der New Economic Policy in der zweiten Phase des Entwicklungsweges noch verstärkte, und die nicht wie in vielen Periphieriegesellschaften mit einem intersektoralen Ressourcentransfer vom Agrar- in den Industriesektor verbunden war, sondern vielmehr redistributiv wirkte, implizierte nicht nur das ökonomische Ziel des Wachstums und der Kommerzialisierung der Produktion, d. h. der Marktintegration der Produzenten, sondern gleichzeitig auch das politische Ziel der sozialen Integration der vorwiegend malayischen Bauern. Ihre Systemintegration und -loyalität sowie die Minderung herrschaftsbedrohenden Konfliktpotentials konnte der malaysische Staat bisher recht erfolgreich bewerkstelligen, was durch die relative Stabilität der malaysischen Herrschaftsstruktur unter Beweis gestellt wird.

Die Legitimationsfähigkeit der bestehenden soziopolitischen Struktur wird entscheidend von den Widersprüchen der als Reaktion auf die Rassenunruhen des Jahres 1969 als Integrationsinstrument konzipierten New Economic Policy bedroht. Die wachsenden Disparitäten zwischen städtischen und ländlichen Einkommen sowie eine permanente Verschlechterung der Einkommensverteilung sind ein deutliches Indiz dafür, daß die Nutzeffekte der NEP und speziell der Verteilungspolitik in überwiegendem Maße in den städtisch-industriellen Sektor fließen und sich dort auf eine schmale Elite konzentrieren, d. h. die malayische Staatsklasse, die die NEP für ihre eigenen Interessen funktionalisiert.

Die Faktoren einer instabilen Entwicklung der Realeinkommen, denn der positive Trend kann jederzeit durch ein Sinken der Weltmarktpreise sowie eine inflationäre Entwicklung der Produktionskosten wieder umgekehrt werden, des strukturellen Ausschlusses von der Verteilungspolitik bei gleichzeitig durch die Proklamation der politischen Ziele der NEP geweckten Erwartungen unter den malayischen Bauern, die durch die Betonung des ethnischen Bewußtseins noch verstärkt werden, bilden jedoch ein breites soziales Konfliktfeld. Die Labilität der Situation läßt sich deutlich am Protest der Reisbauern in Kedah gegen ein von der Regierung verordnetes Zwangssparen von 2 M\$ des Reispreises ablesen, die mit ihren Demonstrationen gezeigt haben, daß sie eine Verschlechterung ihrer Einkommen nicht widerstandslos hinnehmen.

Aber nicht die Ärmsten der Armen, sondern die privilegierten Bauern des MUDA-Schemes gingen hier auf die Straße, um ihre Interessen gegenüber dem Staat zu verteidigen. Bisher garantierten ein hohes Wirtschaftswachstum ${ }^{17}$ sowie die Einnahmen aus der Differentialrente der Erdölsteuern der malayischen Staatsklasse jedoch einen ausreichenden finanziellen Handlungsspielraum, um sowohl auf die Ansprüche der Bauern reagieren als auch ihre Eigeninteressen durchsetzen zu können und insgesamt trotz der offensichtlichen Widersprüchlichkeiten der Agrarpolitik die soziale Integration der Bauern zu gewährleisten.

17 Nach den jüngsten Prognosen von Chase Econometrics, einer Tochterfirma der New Yorker Chase Manhat$\tan$ Bank, wird Malaysia in den achtziger Jahren die höchsten Wachstumsraten auf weisen können: "Malaysia will emerge as the new Asian model of high growth with low inflation"; vgl. FEER, v. 8. 5. 1981. 


\section{Literaturverzeichnis}

Barlow, C. 1978, The Natural Rubber Industry. Its Development, Technology, and Economy in Malaysia, Kuala Lumpur.

Bell. C./Hazell 1980, Measuring the Indirect Effects of an Agricultural Project in its Surrounding Region; in: American Journal of Agricultural Economics, Vol. 62, No. 1, Feb. 1980, pp. 75-86.

Bode, T./Müller-Debus, T.E. 1978, Direktinvestitionen in Malaysia. Der Einfluß auf die wirtschaftliche Entwicklung, Tübingen.

Elsenhans, H. 1976, Zur Rolle der Staatskasse bei der Uberwindung der Unterentwicklung; in: Schmidt, A. (Hg.) Strategien gegen Unterentwicklung. Zwischen Weltmarkt und Eigenständigkeit, Frankfurt/M. pp. 250-265.

Evers, H. D. 1978, On the Evolution of Urban Society in Malaysia, Manuskript, Bielefeld.

Fredericks, L. J. 1977, Ideology and Organization in Agricultural Development: The Case of Malaysia; in: Sociologia Ruralis, Vol. XVII, No. 3, 1977, pp. 191-202.

-/Wells, R. J. G. 1978, Some Aspects of Tenancy Reform Measures in Southeast Asia; in: Asian Survey, Vol. XVIII, No. 6, June 1978, pp. 644-658.

Fu-Chen Lo/Salih, K. 1977, Regional Policy and Urban Transformation in Asi UNCRD, Nagoya/Japan.

Galenson, A. 1980, Agriculture and Rural Poverty; in Young, K. et a Malaysia. Growth and Equity in a Multiracial Söciety, Baltimore/London, pp. 211-252.

Goldman, R. H. 1975, Staple Food Self-Sufficiency and Distributive Impact of Malaysian Rice Policy; reprinted from Food Research Institute Studies, Stanford University, California, USA, Vol. XIV, No. 3, 1975, pp. 251-293.

Griffin, K. u. Mitarbeit v. Ghose, A. K. 1979, Growth and Improverishment in Rural Areas of Asia; in: World Development, Vol. 7, 1979, pp. 361-383.

Grilli, E. R. et al 1978, The World Rubber Economy: Structure, Changes, Prospects, World Bank/FAO, Washington D. C.

Hanisch, R./Tetzlaff, R. (Hg.) 1981, Staat und Entwicklung. Studien zum Verhältnis von Herrschaft und Gesellschaft in Entwicklungsländern, Frankfurt/M.

IBRD/FAO 1975, Problems of Rural Poverty in Malaysia, Rome.

Jägeler, F.J. 1976, Natürliche Rohstoffe im Substitutionswettbewerb. Das Beispiel des Naturkautschuks, Hamburg.

Jegathesan. S. 1977, The Green Revolution and the MUDA Irrigation Scheme. An Analysis of its Impact on Size, Structure and Distribution of Rice Farmers Income, MADA Monograph No. 30, Alor Setar/Kedah.

Jomo, K. S. 1978, Spontaneity and Planning in Class Formation: The Ascendancy of the Bureaucratic Bourgeoisie in Malaysia. Paper presented at the IX. World Congress of Sociology, Uppsala/Sweden.

Kasch, V. 1981, Kautschukabkommen: Ein Sonderfall? in: epd-Entwicklungspolitik 8/81, pp. 15-16.

- - 1982, Agrarpolitik in Malaysia. Zur Rolle des Staates im Entwicklungsprozeß, Diss. Hamburg.

Kessler, C. 1978, Islam and Politics in a Malay State. Kelantan 1838-1969, Ithaca/London.

Khoo Khay Jin 1978, The Marketing of Smallholder's Rubber, UNCRD, Nagoya/Japan.

Koninck, $R$. De 1979, The Integration of the Peasantry: Examples from Malaysia and Indonesia; in: Pacific Affairs, Vol. 52, No. 2, Summer 1979, pp. 265-293.

Lee, E. 1977, Rural Poverty in West-Malaysia, 1957-70; in: ILO, Poverty and Landlessness in Rural Asia, Geneva, pp. 185-203.

Lim Sow Ching 1976, Towards an Equitable International Trade in Natural Rubber; in: Malaysian Rubber Review, Vol. 1, No. 1, July 1976, pp. 13-24.

Lim Teck Ghee 1977, Peasants and their Agricultural Economy in Colonial Malaya 1874-1941, Kuala Lumpur.

Ouchi, T. et al. 1977, Farmer and Village in West-Malaysia, Tokyo.

Rabenau, K. v. 1977, Entwicklungstendenzen der Einkommensverteilung in Entwicklungsländern: Das Beispiel Malaysia, Regensburg.

Robertson, A. F. 1975, A New Kind of Malaysian; in: Journal of Administration Overseas, Vol. 14, 1975, pp. 30-38.

Rokiah. A. Talib 1978, Two Models of Land Development. Malaysian Experience. Paper presented at the Xth International Congress of Anthropological and Ethnological Sciences, New Dehli.

Selvadurai, S. 1978, Agriculture in Peninsular Malaysia. Bahagian Perancang Dan Pembanguan Kementerian Pertanian, Kuala Lumpur.

Snodgrass, D. R. 1980, Inequality and Economic Development in Malaysia, Kuala Lumpur.

Vorys, K. v. 1975, Democracy with out Consensus, London. 


\title{
Guyana's new Constitution and the elections of 1980: A case of people's power?
}

\author{
by Rajendra Chandisingh
}

The purpose of the essay is to examine Guyana's new Constitution with the object of assessing whether the claims of the PNC government can be justified that it is a socialist constitution necessary for the government's much vaunted socialist revolution and that it offers real power to the broad masses for participating effectively in the important decision-making processes in Guyana's political and economic life. A detailed analysis of the provisions of the Constitution shows, however, that while the basic principles and the fundamental political, social and economic rights may be praiseworthy, the powers, immunities and protection of the executive President are so all-embracing that they cannot accord with any reasonable assumptions one may make about socialist democracy and "people's power". In addition, the "organs of local democratic power", should they fall into the wrong hands, offer an effective mechanism for the execution of efficient and total control of the whole population.

In the context of the concrete political situation in Guyana, however, the Constitution takes on a more sinister look. Evidence is given of the PNC's denial of civil rights and of its repressive methods of rule. The elections based on the Constitution which the PNC won with a massive majority was only the latest in a series of rigged elections going back to 1968. The inescapable impression is that the Constitution has little to do with socialism and socialist democracy and a great deal to do with providing constitutional legality for what had already de facto existed, viz. a harsh, undemocratic regime intent on maintaining power.

\section{State and Agricultural Sector in West-Malaysia}

by Volker Kasch

The second phase of the Malaysian development strategy started with the concept of the New Economic Policy, which is characterized by a significant change of state functions. Public policy is now carried out by a capitalistic oriented state class. While she is trying to create her own economic base by expanding the public sector in industry her agricultural policy persuing the aim of reradication of poverty' is essentially directed towards securing political legitimacy amongst the Malayan peasantry, i. e. the agricultural policy serves at the same time an economical and political function.

Because of on the one hand existing economical and political interests and on the other hand comparative cost advantages as well as the existence of an enormous land potential the agricultural export sector formed the leading sector' within the Malaysian develop- 
ment strategy being world market oriented. Accordingly a major proportion of the public development expenditures went into the agricultural sector and only a minor part into the industrial sector in contrast to the experience of many underdeveloped countries. The agricultural policy concentrated on three areas: land settlement, modernization (rubber) and diversification (palm oil) of the export sector and importsubstitution of rice. Significant features of the agricultural policy are the centralized and bureaucratic character of the numerous public Development Authorities, the lack of every institutional reform and chances of participation of the peasants (reform from above) and the concentration on capital intensive development projects and the relative neglect of the in-situ-developmentr. This constitutes the very contradiction of the policy of the Malayan state class. On the economical level the world market dependent agrarian strategy was quite successful and did not lead to an absolute expansion of mass poverty nor the marginilization of disposessed farmers. However, the creation of a middle class peasantry and the structural exclusion of a large group of poor peasants from the modernization programmes of the state who are not able to accumulate because of the tinyness of their holdings has developed a process of social polarization. The obvious deterioration of the income distribution, the expansion of the sectoral and regional income disparities including the instability of the world market dependent real incomes imply a major conflict potential that threatens the legitimacy and therefore the political position of the Malayan state class.

\section{The significance of "promoting function" for co-operatives in Developing Countries}

\section{by D. J.M. Soulas de Russel}

The co-operative societies in Developing Countries have to understand their promoting function in a different way as in Europe. First of all, they must take more distant standing vis-à-vis their economic dimension, as they do in industrial Countries, and they will have to practice a much more intensive solidarity in all their socio-cultural activitiess. The co-operative in Developing Countries is primarily threatened in its autonomous status by the government, which of ten attemps to manipulate it ("officialization"). When they succeed in relaying the autochthones forms of co-operation, the modern co-operatives would be able to introduce ("vehicular function") appreciable improvements in economic and human fields ("function of humanization"). Those opposed to these purposes can only be overthrown with the government's and the international community's support. At least, the co-operatives' viability in the Third World depends on their own dimension (controlability), on the actual adaptation of their legal structures and on the andragogical capabilities of their staff members (multidisciplinal and creative training). 